\title{
Review on the book Anatoly Zhigljavsky, Antanas Žilinskas "Bayesian and High-Dimensional Global Optimization", SpringerBriefs in Optimization, Springer, 2021
}

\author{
Yaroslav D. Sergeyev ${ }^{1,2}$
}

Received: 28 June 2021 / Accepted: 7 July 2021 / Published online: 14 July 2021

(c) The Author(s) 2021

Global optimization (GO) is a broad and active field of research including development of algorithms for solving discrete and continuous problems, their theoretical analysis, and applications to various real-word problems. The book under review is mostly devoted to some methodological and theoretical issues of continuous GO with an emphasis on Bayesian and high-dimensional GO algorithms. In the optimization literature, an increasing interest to these topics can be observed during the last few decades. The development of new algorithms was stimulated by appearance of applications arising especially in computer experiments, machine learning and optimal engineering design where optimization problems are often characterized as black-box and expensive. In the present monograph, the theoretical investigation in Bayesian and high-dimensional GO was carried out in parallel with the development of algorithms; many challenges and unsolved problems are also discussed. The book is written by two top researchers in the field of stochastic GO who have been awarded a prestigious Constantin Carathéodory Prize by the International Society for Global Optimization (Antanas Žilinskas in 2017; Anatoly Zhigljavsky in 2019), both for their life-time achievements in stochastic global optimization.

The book consists of three chapters. The first one is devoted to the problem of space-filling in high-dimensional sets, which constitutes a very important part of many GO algorithms. The authors discuss various aspects of uniformity and spacefilling and demonstrate that good uniformity of a set of points is by no means implying its good space-filling. In this chapter, space-filling of points is considered from the viewpoint of covering and weak covering, where only a large part of a given set has to be covered by the balls with centres at the chosen points rather than the full set, as in the standard covering. Many interesting geometrical features of highdimensional sets are considered and well-illustrated. In fact, Chapter 1 contains 24 figures, some of which are rather informative and attractive. Another interesting

Yaroslav D. Sergeyev

yaro@dimes.unical.it

1 University of Calabria, Rende, Italy

2 Lobachevsky State University, Nizhni Novgorod, Russia 
feature of presentation of this chapter is formulation and discussion of several conjectures, with some of them well-known (like the Riemann hypothesis) and some other being original.

Chapter 2 deals with Bayesian GO, which recently became one of the most actively studied directions in non-convex optimization. The Bayesian approach to GO has attracted great interest due to successful applications in machine learning, optimal engineering design, and many other areas. The authors of the book discuss the development of this approach starting with the original ideas of the algorithms optimal in the sense of the expected approximation error, and of the expected improvement. The theoretical and implementation challenges are discussed in conjunction with advantages and successful applications. The poorly researched problem of the selecting and estimating parameters of the probabilistic model of the objective function is analyzed and several practical recommendations are formulated. The old problem about the theoretical basis of the one-step optimal algorithms is analyzed from the point of view of the rational decision theory. As a result of this analysis, the bi-objective selection of the current search step is justified formalizing the desirable compromise of the criteria of exploitation and exploration. The inner computational complexity of the Bayesian GO algorithms significantly limits their applicability. As shown in the book, the mentioned complexity can be circumvented by the partition-based implementation. The chapter is completed by the analysis of the convergence of the considered Bayesian GO algorithms and the examples of their applications. The pros and cons of the Bayesian GO are discussed. The careful analysis of challenges is especially welcome since most recent publications present only the results of successful applications of the Bayesian algorithms.

Chapter 3 reviews the subject of the global random search in continuous GO, mostly keeping in mind the use of such algorithms in reasonably large dimensions. In this chapter, many recent results are discussed along with selected well-known and rather old results. The main attention is paid to construction of efficient statistical inference procedures about the global minimizer and to the population-based algorithms.

The book is written for a wide circle of readers and will hopefully be appreciated by those interested in theoretical aspects of global optimization as well as practitioners interested mostly in the methodology. All those interested in applications of global optimization can also benefit from the book. All in all, this book is an excellent addition to the existing literature on global optimization and gives one more evidence that the subject of global optimization is very much alive.

Funding Open access funding provided by Università della Calabria within the CRUI-CARE Agreement.

Open Access This article is licensed under a Creative Commons Attribution 4.0 International License, which permits use, sharing, adaptation, distribution and reproduction in any medium or format, as long as you give appropriate credit to the original author(s) and the source, provide a link to the Creative Commons licence, and indicate if changes were made. The images or other third party material in this article are included in the article's Creative Commons licence, unless indicated otherwise in a 
credit line to the material. If material is not included in the article's Creative Commons licence and your intended use is not permitted by statutory regulation or exceeds the permitted use, you will need to obtain permission directly from the copyright holder. To view a copy of this licence, visit http:// creativecommons.org/licenses/by/4.0/.

Publisher's Note Springer Nature remains neutral with regard to jurisdictional claims in published maps and institutional affiliations. 